\title{
Comparative study of three methods of plastic embedding in diagnostic dermatopathology
}

\author{
MARY MASON, RONA M MACKIE \\ From the Department of Dermatology, University of Glasgow, Glasgow
}

SUMMARY Three methods of plastic embedding were assessed for their value in routine dermatopathology. The J B 4, Taab embedding, and Taab transmit techniques were compared for quality, convenience, and safety. The Taab transmit method was the most satisfactory method, being both rapid and simple. An additional advantage is that the resin can be removed and the immunoperoxidase technique carried out on thin sections.

Techniques for plastic embedding are now routinely used in many pathological specialities, including haematology and renal pathology. ${ }^{1-4}$ The principle advantage is greater resolution at higher magnifications using the light microscope. This property, on occasion, permits the visualisation of structure or of cellular detail which previously required use of the electron microscope.

Techniques for plastic embedding have not yet become widespread in routine dermatopathology despite their obvious advantages in accurately identifying such features as the hyperconvoluted nuclei of the atypical lymphocytes in mycosis fungoides and intraepidermal cells with and without desmosomal attachments. It is well known that techniques that are entirely satisfactory applied to tissue such as lymph node may not be optimal for skin because of the varying permeability of the dermis and epidermis. For this reason we studied the results obtained with three different embedding techniques in normal and abnormal skin samples. We also investigated the suitability of each of these three methods for subsequent immunopathological techniques, using monoclonal antibodies and the immunoperoxidase technique.

\section{Material and methods}

Samples of human skin up to $1 \times 0.5 \times 0.5 \mathrm{~cm}$ in size were fixed in formol calcium for 24 hours and then processed individually in bijoux containing the appropriate solution.

\section{METHOD 1: J B 4 (2-HYDROXYETHYL}

METHACR YLATE)

After fixation the sample was dehydrated in three

Accepted for publication 21 August 1985 changes of 2,2 dimethoxypropane (DMP) (1 drop concentrated hydrochloric acid/100 ml DMP) for 40 minutes in each change and two twenty minute changes in propylene oxide. The sample was then impregnated by placing in $\mathrm{J}$ B 4 processing solution (glycol methacrylate $80 \mathrm{ml}$ and benzoyl peroxide 1 $\mathrm{Gm}$ ) for two hours followed by J B 4 embedding solution (J B 4 processing solution plus $1 \mathrm{ml}$ polyethylene glycol) for two hours. Polymerising solution $(4.6 \mathrm{ml}$ embedding solution plus 1 drop $\mathrm{N}-\mathrm{N}$ dimethylaniline) was then added to the sample in a small mould, and the mould was covered and left overnight.

\section{METHOD 2: TAAB EMBEDDING RESIN}

The sample was dehydrated, as previously described, using acidified DMP and propylene oxide and impregnated in a 1:1 solution of propylene oxide and Taab resin mix for two to four hours followed by resin mix only for 24 hours. The sample was finally embedded in fresh resin in a small mould for 24 hours at $60^{\circ} \mathrm{C}$. Taab resin mix is composed of $10 \mathrm{ml}$ of Taab resin, $10 \mathrm{ml}$ of Taab hardener, and $0.4 \mathrm{ml}$ benzyl dimethylamine (Taab Laboratories Ltd, Reading, Berkshire).

\section{METHOD 3: TAAB TRANSMIT RESIN}

After dehydration the sample was impregnated in a 1:1 solution of propylene oxide and resin mix for one hour and then in a solution of Taab resin mix for three to four hours. Polymerisation was then achieved by embedding the sample in a mould containing fresh resin for $12-18$ hours at $60^{\circ} \mathrm{C}$. The solution used comprised $6.5 \mathrm{ml}$ Taab transmit resin and $18 \mathrm{ml}$ Taab hardener TH1, which was mixed and left for 30 minutes when $0.5 \mathrm{ml}$ transmit accelerator was added. Cutting and staining details for all methods After preparation the tissue sample was attached to a 
wooden chuck with Loctite superglue. The sample was cut at a thickness of 1-2 $\mu \mathrm{m}$ on a Jung autocut microtome with a Ralph glass knife. Sections were cut dry. For method 1 the sections were laid on a water bath at $37^{\circ} \mathrm{C}$, mounted on a glass slide, dried on a hot plate for one hour, and further dried in the oven at $70^{\circ} \mathrm{C}$ for one hour. For methods 2 and 3 sections were placed on a slide which was then flooded with absolute alcohol. Sections were then floated on to a water bath and mounted and dried as for method 1 .

Sections obtained using all methods were then stained with routine haematoxylin and eosin, Giemsa, periodic acid Schiff, elastica, Dominici, ${ }^{56}$ and Huber ${ }^{7}$ stains. Sections prepared using methods 2 and 3 had the resin removed by placing in a saturated solution of sodium hydroxide in absolute alcohol for 30 minutes. The routine immunoperoxidase technique could then be applied to these sections.

Ninety samples of clinically and histologically normal skin were studied using these three methods. These samples were obtained during routine plastic surgery procedures. A further 13 samples of abnormal skin were studied by all three methods.

\section{Results}

Careful comparison of the advantages and disadvantages of these three methods showed that the Taab transmit resin technique was the most satisfactory. The processing procedure could be completed in one

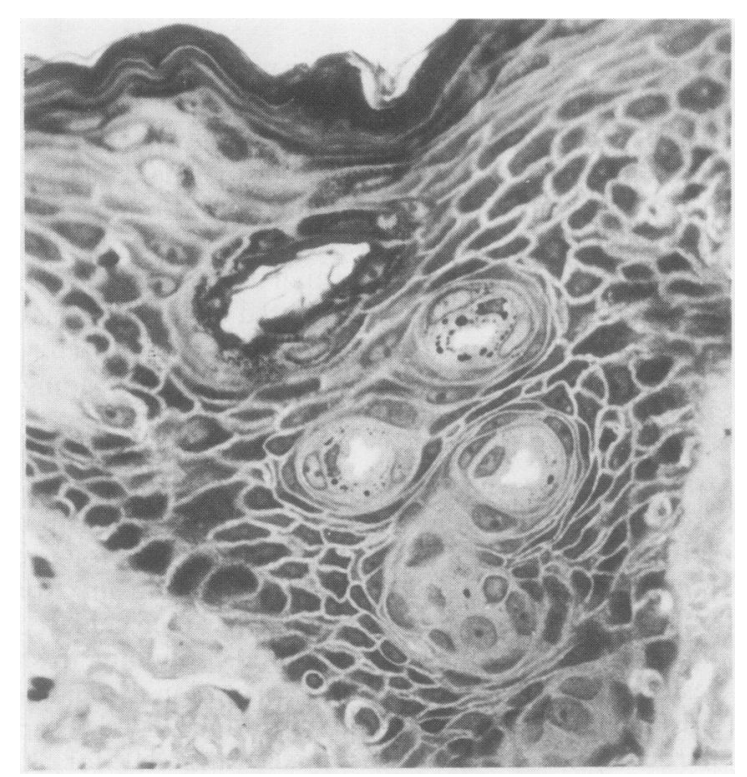

Fig. 1 Normal human epidermis clearly showing intraepidermal portion of eccrine sweat duct. (Haematoxylin and eosin.) day, there were few problems with flotation of sections, and the resin could be removed to permit use of immunopathological techniques. Figs 1 and 2 illustrate the quality of preparation obtained.

The J B 4 (2-hydroxyethyl methacrylate) method produced high quality stained preparations and could also be completed in one day. The materials can be purchased in kit form, which is convenient but expensive. Throughout this study we made up our own materials and this was not unduly time consuming.

The main disadvantages of this method are that some dyes do not penetrate the plastic and therefore the choice of stains is limited and that immunopathological techniques cannot be performed on the sections as the resin cannot be removed. Some published reports of this technique use an overnight polymerisation, thus lengthening the processing time.

We found that the Taab embedding technique was comparatively inefficient. The method took 2 days, and there were continual problems with flotation of sections. Sections also tended to disintegrate during the staining process.

We found no particular advantages in using any of the special stains advocated by other groups compared with conventional haematoxylin and eosin staining.

Using a panel of monoclonal antibodies after removal of the resin, we found that certain antibodies gave some staining. These were Campath $5 \cdot 2$ ?

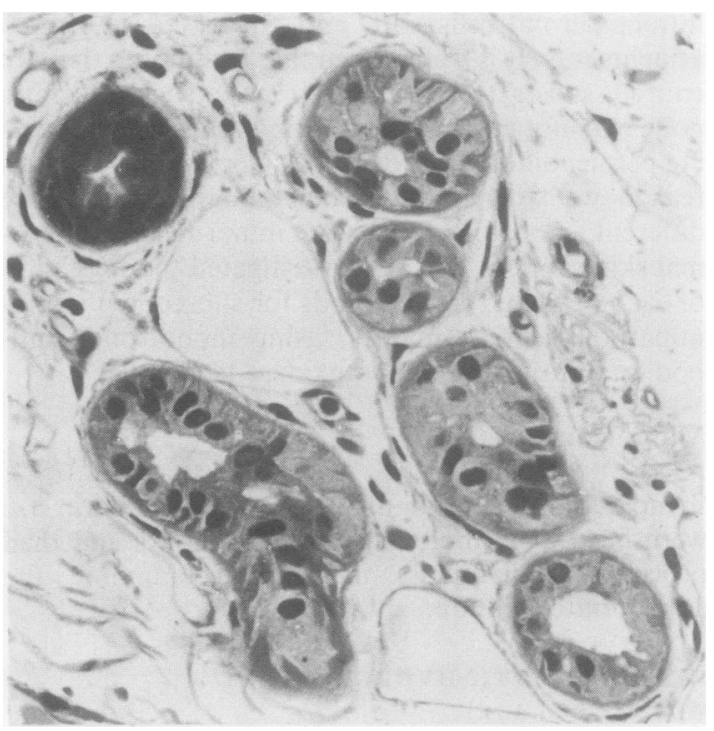

Fig. 2 Normal human dermis showing secretory and excretory coils of eccrine sweat duct. Different cell types found in these structures are clearly visualised. 
(cytokeratin $^{8}$ ), NKI C3 ${ }^{9}$ (recognises benign and malignant melanocytic cells), and antibody to common leucocyte antigen (Dako CLA), which recognises all cells of the leucocyte series. The pan $T$ and helper and suppressor lymphocyte surface markers did not stain.

\section{Discussion}

The results of this technical study clearly showed the superiority of the Taab transmit method for routine dermatopathological use. This is a useful observation as previous workers have advocated the J B 4 method for routine dermatopathological use. ${ }^{10}$ These studies, however, did not include preliminary assessment of the relative merits of different techniques; if we had proceeded with this method without the preliminary survey we would have used a method twice as time consuming as the Taab transmit method and would have had the additional disadvantage of using a resin that is not removable for immunoperoxidase studies.

Unsurprisingly, the delicate lymphocyte membrane surface markers were not preserved in tissue embedded in resin as this staining is also lost with routine processing, and frozen sections are required. The fact that some antigenic activity was preserved with keratins, cells of the melanocyte series, and leucocytes is encouraging with regard to future use of thin sections and immunopathologial studies in combination.

As a result of this preliminary survey we have started a study to assess the value of thin sections in the histological analysis of cutaneous lymphoid infiltrates.
We gratefully acknowledge financial assistance from the Skin Disease Research Fund.

\section{References}

${ }^{1}$ Aparichio SR, Marsden P. Application of standard microanatomical staining to assess epoxy resin-embedded sections. J Clin Pathol 1969;22:589-92.

${ }^{2}$ Eastham WN, Essex WB. Use of tissue embedded in epoxy resin for routine histological examination of renal biopsies. J Clin Pathol 1969;22:99-106.

${ }^{3}$ Chi EY, Smuckler EA. A rapid method for processing liver biopsy specimens for 2 micron sectioning. Archives of Pathology 1976;100:457-62.

${ }^{4}$ Afroudakis AP, Liew CT, Peters RL. An immunoperoxidase technique for the demonstration of the hepatitis $B$ surface antigen in human liver. Am J Clin Pathol 1976;65:533-9.

${ }^{5}$ Green GH, Kurrein F. Glycol methacrylate embedding in general pathology. Association of Clinical Pathologists Broadsheet No 97, 1981.

${ }^{6}$ Gray P. The microtomists formulary and guide. New York: Blakiston Co, 1954.

${ }^{7}$ Huber JD, Parker F, Odland GF. A basic fuchsin and alkalinized methylene blue rapid stain for epoxy embedded tissue. Stain Technology 1968;43:83-7.

${ }^{8}$ Makin CA, Bobrow LG, Bodmer WF. Monoclonal antibody to cytokeratin for use in routine histopathology. J Clin Pathol 1984;37:975-84.

${ }^{9}$ MacKie RM, Campbell I, Turbitt ML. Use of NKI C3 monoclonal antibody in the assessment of benign and malignant melanocytic lesions. J Clin Pathol 1984;37:367-72.

${ }^{10}$ Saint Agnese P, Jensen KL. Dibasic staining of large epoxy tissue sections and applications to surgical pathology. Am J Clin Pathol 1984;81:25-9.

Requests for reprints to: Professor Rona M MacKie, Department of Dermatology, Anderson College Building, 56 Dumbarton Road, Glasgow E11 6NU, Scotland. 